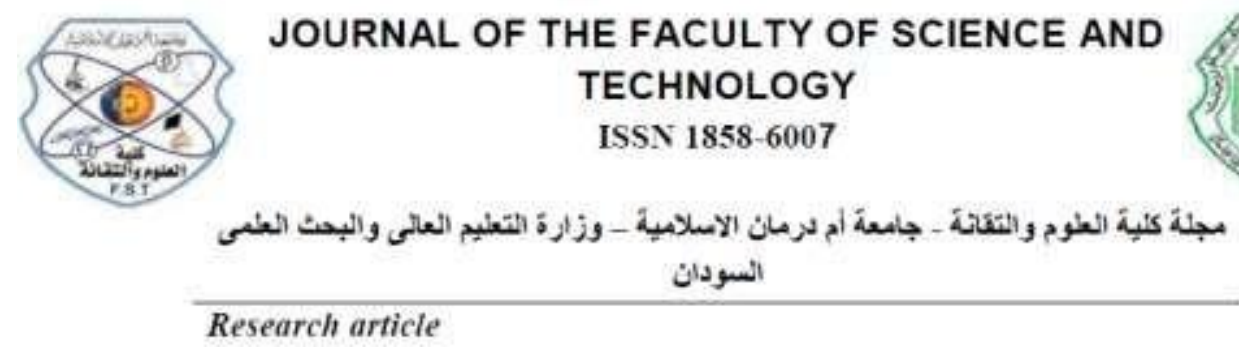

\title{
Histopathologic Changes in Liver Tissues From Male Albino Rats Treated with Croton tiglium Mixed With Animals Diet
}

\author{
Awatif M.E Omran ${ }^{1,2}$, Salah A.M Ali ${ }^{1}$, Elkamali H.H ${ }^{3}$ \\ ${ }^{1}$ Dept. of Biotechnology faculty of Science and Technology, Omdurman Islamic University. \\ Omdurman, Sudan. \\ ${ }^{2}$ Dept. of Biochemistry, College of Science, Tabuk University \\ ${ }^{3}$ Dept. of Botany faculty of Science and Technology, Omdurman Islamic University, Omdurman, \\ Sudan.
}

\section{Awatify2006@gmail.com}

\begin{abstract}
Croton tiglium is commonly used to treat constipation in African countries including Sudan. This study aim to evaluate histopathologic changes in Liver tissue after treated with Croton tiglium mixed with animals diet in male albino rats. The rats were divided into three groups containing of 6 rats per group for each, and they treated as follow : Group I serve as control, Group II \& III: Were given a mixture of animals diet with Croton tiglium diet at concentrations of $10 \%$ and $20 \%$ respectively. Treatment of rats with $10 \%$ of Croton tiglium showed no remarkable damages or signs of lesions on specimens taken from animals for histopathological examinations ,but rats under $20 \%$ treatment showed some abnormal (alterations) .Therefore, the fruits of Croton tiglium at $20 \%$ are toxic to rats .

Further studies are necessary to isolate and characterize the bioactive constituents in the fruits and to elucidate modes of compound actions and interactions.
\end{abstract}

Key words; Croton Tiglium, Histopathologic Changes ,Albino rats. 


\section{INTRODUCTION}

\section{Test Animals:}

Eighteen adult albino male rats of age 6-8 weeks with an average body weight 120-160 grams, were used in this study. After acclimatization period, the animals were divided into three groups each of six ,and they treated as follow:

Group I: given normal animals diet and serve as control.

Group II \& III: were given a mixture of animals diet Croton tiglium crushed seeds fed diet as concentrations of $10 \%$ and $20 \%$ respectively.

The experiments were conducted for two weeks. At the end of the experiments the animals were slaughtered.

\section{Histopathological Examination.}

Necropsies were done on all slaughtered rats to identify any growth lesions. Specimens of Liver were fixed in $10 \%$ of neutral formalin.

Embedded in paraffin wax, sectioned at $5 \mu \mathrm{m}$ and stained with heamatoxylin and lesions for histopathological Examination.
Croton Tiglium L , which locally known as (Habat Almulook) is belong to family Enpherobiaceae it is used as laxative, tumors and cancerous sores (salatino et al, 2007).

The seeds yield fatty acids compared principally of stearin , planitin ,glycerids of crotonic and tiglic acids ,the glucoside crotonoside and alkaloids.

It should be used cautiously because of it is high toxicity, should not be administered to pregnant women (parjapat et al, 2010).

This study aim to evaluate histopathologic changes in Liver tissues after treated with Croton tiglium mixed with animals at concentrations $10 \%$ and $20 \%$ in albino rats .

\section{MATERIALS AND METHODS}

\section{Plant Material}

Ripe dried fruits of Croton tiglium used in this study. The fruits were purchased from Omdurman local market and authenticated by authors , the ripe dried fruit were ground to crush form with mortar and pestle before mixing in the diet of the test animals. 
sinusoids which just originated from such central vein show the debris or foreign material as shown in (Figure 4).

Although C.tiglium is commonly used in Sudanese medicine ,the powder of the seeds in mixed with animal diet (Phoenix daetylifera) as laxative (Elkamali and Khalid ,1996).

Information on toxicological interactions in rats unavailable except the study on evaluation of the effect of C.tiglium seeds mixed with animals diet on plasma and blood parameters in male albino rats (Elkamali et al ,2015) .

The result of the present study indicated that, the C.tiglium $20 \%$ mixed with diet is toxic, but not lethal to rats if fed at $10 \%$ of the diet for two weeks .

In conclusion, the study demonstrated that at concentrations used, the fruits of C.tiglium at $20 \%$ are toxic to rats .

Further studies are necessary to isolate and characterize the bioactive compounds in the fruits and to elucidate modes of compound actims and interactions.

\section{RESULTS AND DISCUSSION}

After two weeks of treatment with two different concentrations of Croton tiglium plant mixture with animals diet in which animals fed a diet containing $10 \%$ or $20 \%$ of C.tiglium.

Treatment of animals with $10 \%$ of C.tiglium showed no remarkable damages or signs of lesions on specimens taken from animals for histopathological examination, but animals under $20 \%$ of treatment showed some histopathological alterations.

The hepatic cells were mostly abnormal. Their cytoplasm were vacuolated and showed signs of degenerations, and also altered nucleus and the architecture of the hepatic sinusoids were also lost as shown in (Figure 2), compared with normal liver tissues (Figure 1).

The hepatic plates appear to be formed of cells whose cells borders were lost and the cords appeared like a syncytium of one cell thick end (Figure 3).

The central veins of the hepatic lobules were mostly dilated. Also the hepatic 


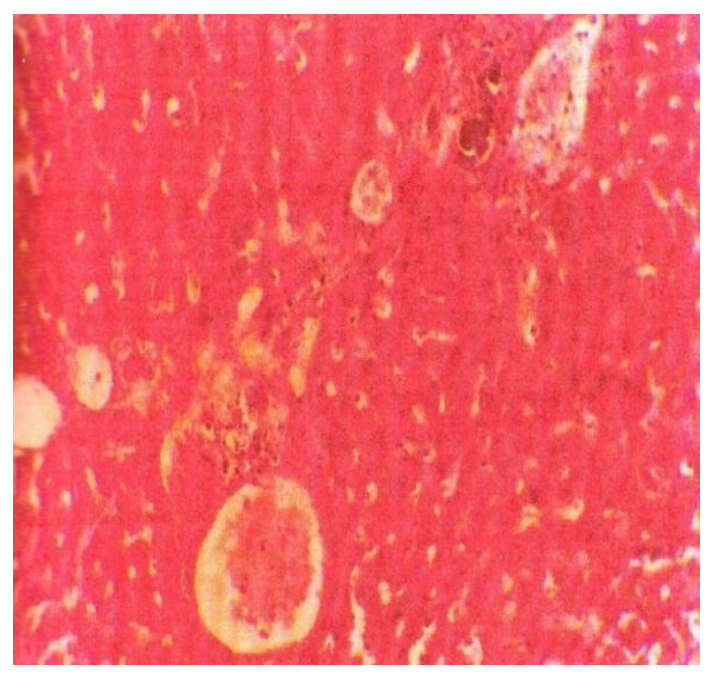

Figure[3] cross section of liver of rats treated with $20 \%$ C. tiglium crushed seeds shows: hepatic plates to be formed of one cell, cell borders lost, hepatic sinusoids engorged with blood, and hepatic veins dilated. $(\mathrm{H} \& \mathrm{E} \times 80)$

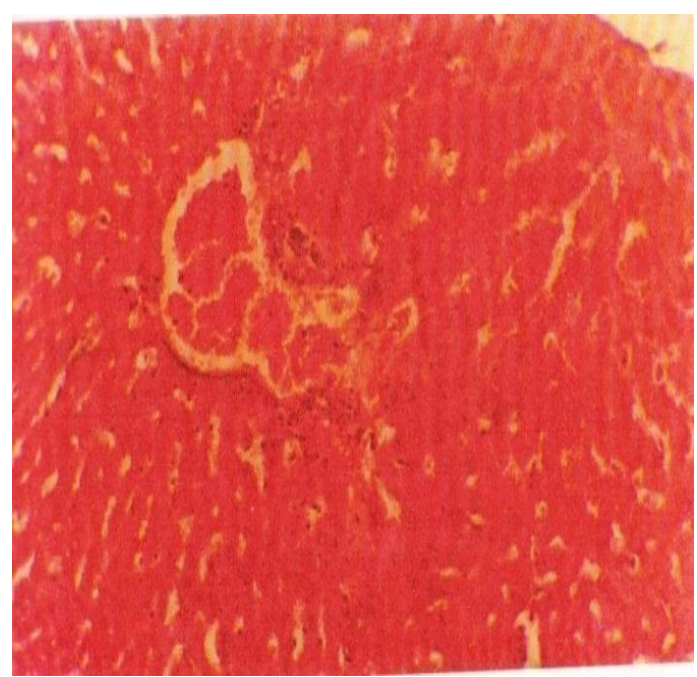

Figure[4] cross section of liver rat treated with $20 \%$ C. tiglium crushed seeds shows: dilated hepatic artery and portal vein, full with homogenous material and some leucocytes. H \& E $\times$ 80

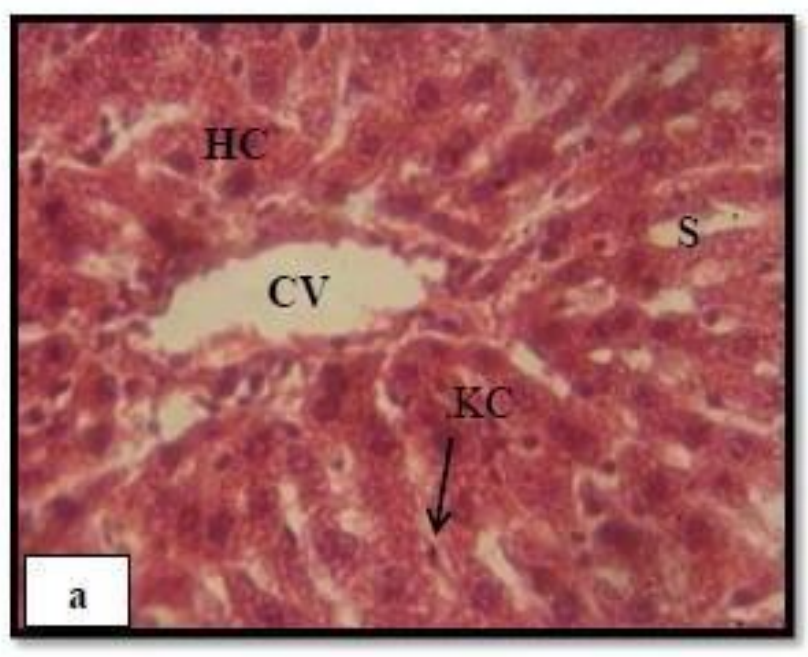

Figure [1] Photomicrographofthe liver sections of mice Control liver showing normal hepatocytes architecture with normal central vein(CV), hepatic cells (HC )sinusoids (S) and Kupffer Cells ( C ).

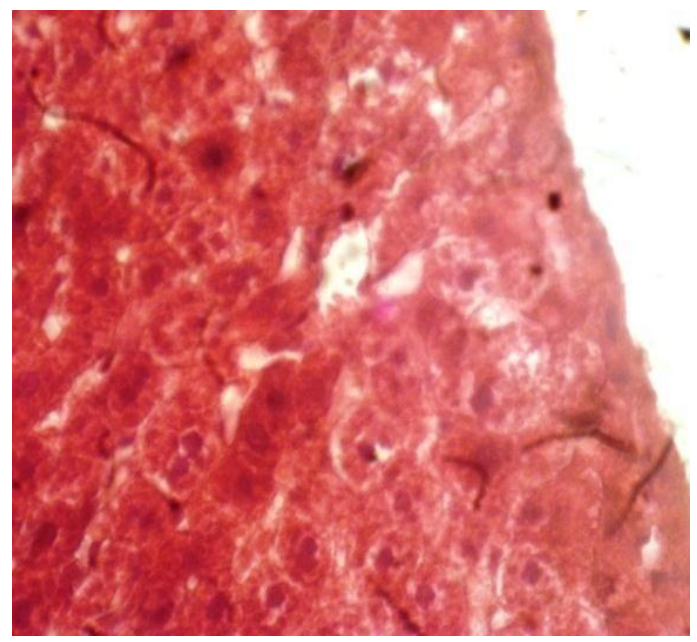

Figure[2] Hepatic cells of liver rat treated with $20 \%$ C. tiglium crushed seeds: shows abnormality signs of degeneration and lost of architecture of the hepatic sinusoids. $\mathrm{H} \& \mathrm{E} \times 80$ 


\section{REFERENCES}

Salatino A.,marials,salatino F.,Negari G.(2007).Traditional Uses ,chemistry and pharmacology of croton species(Euphorbiaceae).T.Baraz.chem soc.18(1):11-33.

Parjapati N.D, Purohit s.s ,shorrmo A.K.,kumer T.(2010). Ahood book of medicinal Plants :A complete source book, Agrobios (India) .page175.
Elkamali HH, Khalid SA(1996).The most herbal remedies in central Sudan Fitoterapia 67(4),303.

Elkamali HH, Omran A.M.E,Abdalla

M.A (2015).biochemical and hematological assessment of croton tiglium seeds mixed with animal diet in male albino rats. Annual Research and Review in Biology 8(4):1-7. 